\title{
Communications
}

\section{Examens de spécialiste \\ Examen de spécialiste en vue de l'obten- tion du titre de spécialiste en chirurgie orthopédique et traumatologie de l'appareil moteur}

Examen de base en chirurgie: Informations: www.basisexamen.ch

Examen final, $1^{r e}$ partie (examen écrit)

Date: Samedi, 6 juin 2015

Lieu: Hôpital de l'Ile, Clinique pédiatrique, auditoire Ettore Rossi, Berne

\section{Examen final, $2^{e}$ partie (examen oral)}

Date: Novembre 2015. La date exacte sera communiquée ultérieurement

Lieu: probablement l'Hôpital cantonal de Fribourg et Swissendos

\section{Délai d'inscription: le 30 avril 2015}

Vous trouverez de plus amples informations sur le site web de l'ISFM www.siwf.ch $\rightarrow$ Domaines spécialisés $\rightarrow$ Titres de spécialiste et formations approfondies (formation postgraduée) $\rightarrow$ Chirurgie orthopédique et Traumatologie de l'appareil moteur

Examen de spécialiste en vue de l'obtention de la formation approfondie en gériatrie à adjoindre au titre de spécialiste en médecine interne générale

Examen écrit:

Date: Jeudi 13 août 2015 (14hoo-17h15)

Lieu: Université de Berne, Hochschulzentrum von Roll, Fabrikstrasse 8, 3012 Berne.

\section{Examen oral pratique:}

Date: Mercredi 28 et jeudi 29 octobre 2015

Lieu: le lieu d'examen est communiqué lorsque les résultats de l'examen écrit sont connus.

\section{Délai d'inscription: le 31 mai 2015}

Vous trouverez de plus amples informations sur le site web de l'ISFM www.siwf.ch $\rightarrow$ Domaines spécialisés $\rightarrow$ Titres de spécialiste et formations approfondies (formation postgraduée) $\rightarrow$ médecine interne générale

\section{Fondation Médecine Sociale et Préventive}

\section{Prix 2015-2016}

Le prix annuel de la Fondation de médecine sociale et préventive, Lausanne, récompense l'auteur d'un travail original de recherche scientifique dans l'un des principaux domaines d'activité de l'Institut universitaire de médecine sociale et préventive (IUMSP) de Lausanne, i.e. épidémiologie et prévention des maladies, évaluation des services de santé.

Le prix est destiné:

- aux assistants réguliers pendant qu'ils occupent une fonction dans un Département, Service ou Institut universitaire ou dans un Hôpital périphérique reconnu pour la formation FMH;

- à tout universitaire effectuant des recherches dans le domaine de la médecine sociale et préventive.

Les candidatures sont examinées par la Commission des Prix et Concours de la Faculté de Biologie et de Médecine. Le prix est décerné lors de la cérémonie de remise des prix de la Faculté de Biologie et de Médecine de l'Université de Lausanne (septembre 2015).

Envoi des dossiers de candidature (indiquant nom, titres, adresse et emploi actuel du candidat): Prix de la Fondation de Médecine Sociale et Préventive, Lausanne, Commission des Prix et Concours de la Faculté de Biologie et de Médecine, Décanat de la Faculté de Biologie et de Médecine, 21, rue du Bugnon 1011 Lausanne.

Délai de dépôt des candidatures: 31 mars 2015

Mach-Gaensslen Stiftung Schweiz

\section{Unterstützung Forschungsprojekt} Neurologie/Psychiatrie

Die Mach-Gaensslen Stiftung Schweiz unterstützt turnusmässig 1-2 Forschungsprojekte der Fachgebiete Kardiologie, Hämatologie/ Onkologie und Neurologie/Psychiatrie. Das Jahr 2015/2016 ist für die Neurologie/Psychiatrie reserviert.

Gesuche um Unterstützung eines qualifizierten Forschungsprojekts des Fachgebiets Neurologie/Psychiatrie können bis spätestens 15. April 2015 an den Präsidenten der Mach-
Gaensslen Stiftung Schweiz gerichtet werden: Prof. Dr. med. Georg Keiser, St.-OswaldsGasse 13, 6300 Zug (gkeiser[at]datazug.ch). Die für das Forschungsgesuch notwendigen Angaben können unter der gleichen Adresse oder telefonisch (o41 71199 75) eingeholt werden. Die Beurteilung der Forschungsprojekte durch den Stiftungsrat erfolgt im Mai 2015.

\section{Schweizerische Gesellschaft für} Neuroradiologie

\section{Peter Huber Preis 2015}

Die Schweizerische Gesellschaft für Neuroradiologie schreibt zum Gedächtnis an Herrn Professor Dr. med. Peter Huber und zur Förderung des Fachgebietes der Neuroradiologie den Peter Huber Preis 2015 aus für eine experimentelle und/oder klinische Arbeit aus dem Gesamtgebiet der Neuroradiologie (allgemeine diagnostische Neuroradiologie, funktionelle Neuroradiologie, interventionelle Neuroradiologie, pädiatrische Neuroradiologie, Neuroradiologie der Kopf- und Halsregion).

Der Erstautor sollte in neuroradiologischer Weiterbildung stehen oder eine neuroradiologische Weiterbildung abgeschlossen haben und nicht über 40 Jahre alt sein. Die eingereichte Arbeit darf 50 Seiten nicht überschreiten und muss in englischer oder deutscher Sprache verfasst sein. Die Arbeit sollte noch nicht ausgezeichnet worden sein und darf nicht an anderer Stelle zur Prämierung eingereicht sein. Pro Person kann nur eine Arbeit eingereicht werden.

Der gestiftete Preis ist mit 2000 Franken dotiert und wird anlässlich der Jahrestagung der Schweizerischen Gesellschaft für Neuroradiologie SGNR am 10.-11. September 2015 in Luzern verliehen. Der Preisträger / die Preisträgerin wird spätestens 2 Wochen vor der Jahrestagung über die Entscheidung des Preiskommitees benachrichtigt und soll die Ergebnisse der Arbeit in einer 8-minütigen Zusammenfassung vorstellen. Der Preis ist teilbar.

Die Arbeiten sind per Mail (mit Abbildungen) bis zum 17. August 2015 an die Geschäftsstelle der Schweizerischen Gesellschaft für Neuroradiologie SGNR, c/o IMK Institut für Medizin und Kommunikation AG, z. Hd. Sandra Leibbrandt, einzureichen (sgnr[at]imk.ch). 